\title{
Conditioning of the rabbit (Oryctolagus cuniculus) nictitating membrane response as a function of trials per session, ISI, and ITI
}

\author{
W. RONALD SALAFIA, W. SCOTT TERRY, and ANTHONY P. DASTON \\ Fairfield University, Fairfield, Connecticut 06430
}

\begin{abstract}
A parametric investigation of nictitating membrane conditioning with interstimulus intervals (ISIs) of 250 or $1,000 \mathrm{msec}$, constant intertrial intervals (ITIs) of 30 or $90 \mathrm{sec}$, and $5,15,30,60$, or 120 trials per daily session revealed that acquisition rate decreased as a function of both larger number of trials per session (NTS) and longer ISI, but with larger overall effects of NTS occurring at the longer ISI. The effects of lengthening the ITI were also greater at the higher NTS and varied as a function of ISI. Results were discussed in terms of several constructs used to explain time-related conditioning effects, namely reactive inhibition, stimulus fluctuation, consolidation, and rehearsal.
\end{abstract}

The number of trials per session (NTS) has received considerable attention in the recent conditioning literature. In a study involving nictitating membrane response (NMR) conditioning, Hupka, Massaro, and Moore (1968) varied interstimulus interval (ISI) and NTS and found that acquisition was directly related to NTS, with faster acquisition at smaller NTS. Moreover, there were only slight differences in acquisition rate as a function of ISI with larger NTS, a finding which seems to be at variance with much of the conditioning literature. Levinthal and Papsdorf (1970), using only one trial per daily session, found a reversal of the usual ISI function. That is, at $1 \mathrm{TS}$, their 1,250-msec ISI group conditioned significantly more rapidly than their 250 -msec group. In a later paper. Levinthal (1973) replicated the earlier finding, and in addition, using 1,5 , or $20 \mathrm{TS}$, found that the ISI reversal took place between 1 and 5 TS. In a more recent study, Kehoe and Gormezano (1974) employed 1. 5, 10, or $50 \mathrm{TS}$, and confirmed the previous findings that acquisition rate varied systematically as a function of NTS.

In addition to these studies, there is increasing evidence in the rabbit conditioning literature that conditioned responses (CRs) often emerge in the early trials-frequently the first trial-of a session and continue at a rate of nearly $100 \%$ even though no CRs had occurred in previous sessions. This phenomenon has been observed frequently in our laboratory and has been noted in several recent studies (e.g., Bruner, 1965. 1966; Hupka et al., 1968). Although Bruner,

This research was supported in part by NSF Grant 003274 . Portions of the study were read at the annual meeting of the Eastern Psychological Association. New York, 1971. The authors thank Robert S. Bartosiak for his assistance in running some of the animals. Requests for reprints should be sent to W. Ronald Salafia, Department of Psychology, Fairfield University, Fairfield, Connecticut 06430 . who referred to it as an "overnight gains effect" (1966, p. 211), offered no explanation, Hupka et al. suggested that the effect might result from over night consolidation of training. Regardless of the explanation, however, it seems likely that the effect would be related to such variables as NTS.

The purpose of the present study was to investigate the relationship between NTS, ISI, and ITI over an extensive range of NTS values.

\section{METHOD \\ Subjects \\ The subjects were 145 naive male and female New Zealand rabbits, 70 to 100 days old at the start of the experiment. They were maintained on ad-lib food and water and housed in light- and climate-controlled quarters.}

\section{Apparatus}

Four rabbits were run concurrently in a sound-attenuated room containing four separate cubicles, serviced independently by control and recording equipment located in an adjoining room. $A$ panel in front of each subject contained two impedence-matched speakers, used to present the auditory conditioned stimulus (CS) and a continuous white noise for masking during the ITI. A four-channel shock source delivered the US to two stainless steel wound-clip electrodes attached circumorbitally.

Immediately prior to each experimental session, animals were placed in Plexiglas restraining boxes. Movements of the nictitating membrane were monitored by a small photoelectric transducer mounted on each subject's head by means of muzzle-like assembly and mechanically coupled to a surgical-silk suture in the membrane. Signals from the transducers were interfaced through a four-channel control unit, then amplified and graphically recorded by a Grass Model SD polygraph, a CR being defined as a pen deflection of at least $1 \mathrm{~mm}$ occurring within the ISI. Similar apparatus and procedures have been described and illustrated in detail by Gormezano (1966).

\section{Procedure}

Twenty-four hours prior to the first experimental session, subjects were prepared by having a surgical-silk loop inserted into the nictitating membrane of the right eye, under local anesthetic (.5\% proparaocaine hydrochloride), and wound-clip electrodes attached circumorbitally, approximately $5 \mathrm{~mm}$ below the inferior eyelid and $5 \mathrm{~mm}$ posterior to the temporal canthus. Next, each 


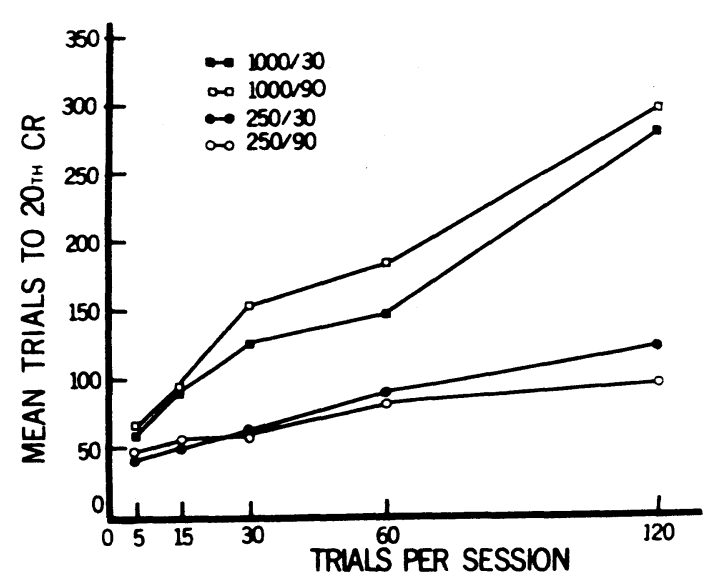

Figure 1. Mean number of triaks to the 20th $C R$ as a function of NTS, for each of the four ISI/ITI combinations.

subject was placed in one of the four cubicles for a 15-min period of adaptation to the cubicle and the 66-dB SPL masking noise.

Conditioning sessions were begun on the following day and continued one session per day until a criterion of $90 \%$ CRs was reached in any block of 20 trials. Because of the large number of animals and long sessions involved in the study, all groups could not be run simultaneously. Therefore, one batch of rabbits was obtained and randomly assigned to each of 10 experimental groups with ISIs of 250 or 1.000 msec. and 5. 15. 30.60. or $120 \mathrm{TS}$. at a 30-sec ITI. After these animals had finished, a new batch was obtained and 10 more experimental groups were formed under the same ISI and TS conditions. but run at a $90-\mathrm{sec}$ ITI. The CS was a continuous $1.000-\mathrm{Hz}$ tone at $85-\mathrm{dB}$ SPL. presented for either 300 or $1.050 \mathrm{msec}$. depending on the ISI. The US was a $3-\mathrm{mA}, 60-\mathrm{Hz}$ shock delivered to the wound-clip electrodes and overlapping the last $50 \mathrm{msec}$ of CS presentation. As each subject reached criterion. it was removed from a cubicle at the end of the criterion session and replaced in the next session by the next subject assigned to the group. This procedure resulted in somewhat uneven $\mathrm{Ns}_{\mathrm{s}}$ with a minimum of five and a maximum of eight subjects in any one group.

\section{RESULTS}

Figure 1 presents the mean number of trials to the 20 th CR for all groups. A 2 by 2 by 5 factorial analysis of variance on these data. adjusted for unequal Ns. revealed that significantly more rapid conditioning took place at the lower NTS regardless of ISI or ITI. $\mathrm{F}(4.125)=32.69 . \mathrm{p}<.001$. Further. conditioning at $250-\mathrm{msec}$ ISI was superior to conditioning at $1.000 \mathrm{msec}$ under all NTS conditions, $F(1,125)=$ 99.20. $\mathrm{p}<.001$. Although there was no significant ITI main effect, $F(1,125)=3.31, p>.05$, there was a signiticant ITI by ISI interaction. $F(1.125)=6.39$. $\mathrm{p}<.025$. indicating that at the higher values of NTS. the 90 -sec ITI produced faster acquisition than the 30 -sec ITI for the $250-\mathrm{msec}$ ISI groups, but slower acquisition for the 1.000 -msec ISI groups. At the smaller NTS. however. there was little difference in conditioning as a function of ITI. There was also a significant ISI by NTS interaction. $F(4.125)=11.87$. $\mathrm{p}<.01$. indicating that the magnitude of ISI-related differences decreased as a function of decreasing VTS.

\section{CR Latency}

Table 1 depicts the mean onset latencies of the first 20 CRs expressed in milliseconds. A progression in the $C R$ latency values is easily seen from lowest values in the upper left end of the table to highest values in the lower right end. A breakdown of this progression shows that CR-onset latency is related to all three variables. with shorter latencies consistently associated with shorter ISI. but also generally associated with shorter ITI and lesser NTS. Because latency variability was also related to ISI. two separate 2 by 5 by 2 mixed factorial analyses of variance were performed to assess NTS- and ITI-related differences, one analysis for each ISI condition. In these analyses. the first factor was ITI. the second was NTS, and the third was first vs. second $10 \mathrm{CRs}$. Both analyses revealed significant ITI main effects. $F(1.68)=$ 22.94. $p<.001$, and $F(1.54)=4.91 . p<.05$, for the $250-\mathrm{msec}$ and $1,000-\mathrm{msec}$ ISI conditions, respectively. That is. for each NTS, the latencies were significantly shorter for the 30-sec ITI groups than for the 90 -sec groups. There was a significant NTS main effect for the 250 -msec ISI. $F(4.68)=8.59 . p<.001$, with latencies decreasing as a function of the lesser NTS. and corresponding trend in the 1.000 -msec groups. although the trend was not statistically significant. $F(4.54)=1.03 . p>.05$. There was also a significant difference between the latencies of the first 10 and the second 10 CRs. $F(1.68)=30.97, p<.001$, and $F(1.54)=7.96, p<.005$, for the 250- and 1.000 -msec groups, respectively, indicating the expected general trend for the CR latencies to become shorter as conditioning trials proceeded. In general. then, it may be seen that shorter latencies accompany those conditions which yield more rapid conditioning. If shortening of CR latency may be taken as evidence for strength of association. then greater associative strength early in conditioning is a function of relatively short ISI and ITI and relatively low NTS.

\section{Intersession Effects}

Since one of the reasons for undertaking the study was to assess intersession gains. a tally was taken of the percentage of animals in each group which emitted the first CR on the first trial of a session. without having emitted CRs previously. The

Table 1

Mean Latencies in Milliseconds of the First 20 CRs as a Function of Trials Per Session

\begin{tabular}{rcccc}
\hline & \multicolumn{4}{c}{ Groups Classified by ISI/ITI } \\
\cline { 2 - 5 } NTS & $250 / 30$ & $250 / 90$ & $1,000 / 30$ & $1,000 / 90$ \\
\hline 5 & 165 & 188 & 490 & 496 \\
15 & 183 & 192 & 477 & 563 \\
30 & 186 & 202 & 487 & 659 \\
60 & 190 & 217 & 525 & 617 \\
120 & 199 & 216 & 563 & 661 \\
Mean & 185 & 203 & 508 & 599 \\
\hline
\end{tabular}


percentages were $50 \%, 35 \%, 30 \%, 22 \%$, and $14 \%$ for the animals conditioned at $5,15,30,60$, and 120 TS. respectively. In addition, many more animals emitted the first $C R$ on the second trial of a session and there was a general tendency in all groups for CRs to emerge in the early trials of a session, rather than toward the middle or end of that session.

\section{DISCUSSION}

The results of the present study are unequivocal in showing a negative monotonic relationship between acquisition rate and NTS. Although the size of the NTS effect differed for different values of ISI and ITI. nevertheless. within each level of these variables. the rate of acquisition of the NMR increased as the NTS decreased. In addition to the NTS effect. there was the predictably large and significant ISI effect. Furthermore, the largest NTS-related differences occurred at the $1.000-\mathrm{msec}$ ISI, with relatively small differences. although consistently in the same direction. at the 2:-0)-msec ISI

The fact that ISI-related differences increased with increasing NTS seemed to differ from the results of Hupka et al. (1968), who found the largest ISI-related differences to be a function of the lower NTS. However, these investigators had analyzed their data in terms of mean percentage of CRs in the first 75 trials rather than trials to the 20th CR or some similar criterion measure. When the present data were evaluated using a similar percentage-CR criterion. they corresponded almost perfectly with those of Hupka et al. That is. the maximum ISI-related differences appeared to be at the lower NTS. However. this method of analysis could be misleading. The apparently smaller ISI-related differences at the larger NTS are actually a function of the minimal amount of conditioning which had taken place over the relatively small number of trials included in the percentage-of-CRs analyses. As the total number of conditioning trials increased. the ISI-related differences increased at the higher rather than the lower NTS. This can best be seen in Figure 1. which depicted the data in terms of trials to the 20th CR. Here. the differences attributable to ISI are clearly greater at the larger NTS.

Lnfortunately. increases in NTS. ISI, and ITI lead. by detinition. to increases in session duration. Visual observation of animals suggested an increased level of arousal and activation associated with both larger NTS and higher ITI. especially at the higher ISI. so that it is possible that one of the major variables involved in differences in performance was the differences in the duration of the various sessions. In addition, it should be mentioned that ITI effects could have been confounded by the fact that all groups were not run simultaneously, as indicated in the procedure. Thus, although the NTS and ISI effects were essentially the same regardless of ITI (as revealed by the combined analysis of the 20 groups presented in the results section, as well as by separate analises of the 10 groups run at each of the ITI values). the ITI effects could have been contaminated by some overall difference in the batches of animals due to their being obtained and run at different times. Although it was deemed necessary to mention this possible source of confounding. previous experience in this laboratory with groups run under the same parameters but at different times has never resulted in a significant difference attributable solely to the different running times.

Analysis of intersession effects revealed that, under all variations of NTS. a relatively high percentage of subjects emitted the first CR on the first trial of a session without having emitted CRs previously, although the percentages varied systematically with NTS Inspection of the individual polygraph records of each animal revealed that the presence or absence of "stepwise learning functions" was also related to NTS. Rabbits frequently go from $0 \%$ CRs to $100 \sigma_{0}$ CRs (or nearly $100 \%$ ) in a single trial, i.e., once the first CR occurs. the animal continues to make only CRs thereafter. This phenomenon will be referred to as stability of conditioning. and thus distinguished from simple variability of performance. Therefore, over a given block of triak, two animals might exhibit the same degree of variability. but one animal's performance be judged as more stable than the other's due to the distribution of LRs and CRs within the trial block. In the present experiment. greater stability of performance, as assessed by the number of animals revealing stepwise learning functions, was also a function of lower NTS, regardless of ISI or ITI, but with the most stable performance occurring as a function of both lower NTS and shorter ISI. That lower NTS leads to more stable conditioning was also noted by Kehoe and Gormezano (1974). who plotted trials to criterion against various criteria from 1 to 10 consecutive CRs. These investigators found that as NTS increased. the number of trials required to attain successive criteria also increased.

From the point of view of conditioning efficiency, the larger NTS clearly led to conditioning in fewer sessions than the smailer NTS. However. a thorough evaluation of efficiency must take into account. not only the number of sessions. but also the total number of trials required. the length of each session. and more importantly, in terms of both methodological and theoretical considerations, the stability of conditioning. The most efficient conditioning in the present study, achieved by a balance of the foregoing considerations. occurred at about 15 to 30 TS. Below 15 TS, a large number of sessions was required. even though conditioning was stable and occurred in the least number of trials. On the other hand, beyond 30 TS. conditioning stability decreased dramatically and the number of trials to criterion increased. although a lesser number of sesions was required for conditioning.

The results of the present experiment were found to be consistent with those of previous NTS studies, although the present study revealed that the basic relationships hold over a wider range of variables and values than were tested previously. In addition. the data indirectly supported the observations of Levinthal and Papsdorf (1970) and Levinthal (1973) with 1 TS. Although 5 TS was the smallest number employed in the present study. the CR criterion data revealed that ISI-related differences diminish with reduction in NTS. Extrapolation of this trend indicates that the ISI-related differences could indeed approach zero and even reverse at 1 or 2 TS.

Choosing from among the major theoretical formulations ordinarily employed to explain time-related conditioning processes is a difficult matter. in light of the many interactions observed in the present study. Although a reactive-inhibition construct (Hull. 1943. 1952) adequately explains the major overall NTS- and ISI-related differences. it is difficult to see how the construct would explain the superior performance of the short-ITI groups over the longer-ITI groups at 1.000 -msec ISI. i.e.. superior performance at the ITI value which would permit less dissipation of inhibition. It is also difficult to see how such a construct would explain the superiority of the longer III at 250 -msec ISI, which should yield. relatively speaking, the lesser amount of inhibition to be dissipated. However. as mentioned previously. these results may reflect performance effects associated with the differences in session duration

A stimulus-fluctuation construct (Estes. 1955) could also handle the basic NTS- and ISI-related differences but, presumably, its explanatory utility decreases as the time periods involved increase. i.e.. the construct applies most directly to events occurring between successive trials which are not spaced too widely apart. Prokasy (1965) suggested that one of the reasons for the ambiguous ITI results to date might be that ITI values tested were too high to tease out the consistent effects. In the present experiment. the ITI values were in a range that would be considered as relatively high.

It is also difficult to see how either of these constructs would account for the intersession-gains effect. since these gains seem to reflect more of an ongoing process of modification or improvement in memory over time, without further overt practice. It appears that a construct such as memory consolidation (Glickman, 1961; McGaugh \& Herz. 1972) more adequately encompasses the intersession gains phenomenon. since it provides a mechanism by which an association between stimuli may become modified or 
consolidated over time, without previous overt CRs having been made. Consolidation was the explanation of choice offered by Hupka et al. (1968) for their NTS data, and moreover, it has frequently been employed to explain the same time-dependent phenomena as the other two constructs.

Finally, for those researchers for whom physiological constructs are less attractive than the more behaviorally oriented ones, Wagner. Rudy, and Whitlow (1973) have recently provided an alternate mechanism. namely "rehearsal." The mechanism appears to be functionally similar to consolidation, and therefore able to handle the NTS and intersession gains effects, although it is behavioral in orientation and it leads to some deductions which are not immediately apparent in the consolidation construct. However, since the rehearsal mechanism has only recently been applied to animal conditioning. further research will be needed to determine its utility.

\section{REFERENCES}

BRUNer. A. UCS properties in classical conditioning of the albino rabbit's nictitating membrane response. Journal of Experimental Psychology. 1965. 69. 186-192.

BRUNER. A. Facilitation of classical conditioning in rabbits by reinforcing brain stimulation. Psychonomic Science, 1966. 6. 211-212.

Estes. W. K. Statistical theory of distributional phenomena in learning. Psychological Review, 1955, 62. 145-154.

Glickman. S. E. Perseverative neural processes and consolidation of the memory trace. Psychological Bulletin, 1961. 58. 218-223.

Gormezano. I. Classical conditioning. In J. B. Sidowski (Ed.),
Experimental methods and instrumentation in psychology. New York: McGraw-Hill. 1966.

Hull. C. L. Principles of behavior. New York: AppletonCentury-Crofts. 1943.

Hull. C. L. A behavior system. New Haven: Yale University Press. 1952.

Hupka, R. B.. Massaro, D. W., \& Moore, J. W. Yoked comparisons of instrumental-avoidance and classical conditioning of the rabbit nictitating membrane response as a function of interstimulus interval and number of trials per day. Psychonomic Science, 1968. 12. 93-94.

KeHOE. E. J., \& Gormezano, I. Effects of trials per session on conditioning of the rabbit's nictitating membrane response. Bulletin of the Psychonomic Society. 1974, 4, 434-436.

Levinthal, C. F. The CS-US interval function in rabbit nictitating membrane response conditioning: Single vs. multiple trials per conditioning session. Learning and Motivation. 1973. 4. 259-267.

Levinthal, C. F., \& Papsdorf, J. D. The classically conditioned nictitating membrane response: The CS-US interval function with one trial per day. Psychonomic Science, 1970, 21, 296-297.

McGaugh, J. L.. \& Herz, M. J. Memony consolidation. San Francisco: Albion, 1972.

Prokasy, W. F. Stimulus fluctuation, reactive inhibition, and time between trials in classical eyelid conditioning. Journal of Experimental Psychology, 1965, 70, 464-472.

WAgner, A. R., Rudy, J. W., \& Whitrow, J. W. Rehearsal in animal conditioning. Journal of Experimental Psychology. 1973. 97. 407-426. (Monograph)

(Received for publication July 21, 1975.) 